\title{
Risco Nutricional versus Risco de Sarcopenia Associado a Complicações Pós-Operatórias e Mortalidade em Pacientes Oncológicos Submetidos a Cirurgias de Grande Porte
}

doi: https://doi.org/10.32635/2176-9745.RBC.2021v67n1.1201

\author{
Nutritional Risk versus Risk of Sarcopenia Associated to Postoperative Complications and Mortality in Cancer Patients \\ Undergoing Major Surgery \\ Riesgo Nutricional versus Riesgo de Sarcopenia Asociado con Complicaciones Postoperatorias y Mortalidad en Pacientes \\ con Cáncer Sometidos a Cirugía Mayor
}

\begin{abstract}
Hadassa Hillary Novaes Pereira Rodrigues'; Maristela Luft Palauro²; Thayse Emanuelli Godoy Behne ${ }^{3}$; Jessika Cadavid Sierra4; Francilene Oliveira Andreo5; Mariana Borges Silva Thé6; José Eduardo de Aguilar-Nascimento ${ }^{7}$; Diana Borges Dock-Nascimento8
\end{abstract}

\section{RESUMO}

Introduçáo: Pacientes cirúrgicos, que apresentam risco nutricional ou de sarcopenia, podem evoluir com piores desfechos no pós-operatório. Objetivo: Investigar se existe associação entre o risco nutricional e a sarcopenia com complicaçôes e mortalidade no pós-operatório de pacientes oncológicos submetidos a cirurgias de grande porte. Método: Estudo bicêntrico de coorte, prospectivo, realizado com 220 pacientes oncológicos adultos, submetidos a operaçóes de grande porte no Hospital de Câncer e na Santa Casa de Misericórdia em Cuiabá, Mato Grosso. Os pacientes foram classificados com ou sem risco nutricional pela Nutritional Risk Screening 2002 e de sarcopenia segundo o questionário Strength, Assistance with walking, Rise from a chair, Climb stairs - and Falls, no pré-operatório. As variáveis de desfecho foram complicações infecciosas e óbito no pós-operatório. Resultados: Os pacientes com risco nutricional mostraram maior risco de complicaçóes infecciosas (24,6 vs. 5,1\%; $\mathrm{RR}=4,8 \mathrm{IC} 95 \%$ 1,94-12; $\mathrm{p}<0,001)$ e de óbito (11,5 vs. 1,0\%; RR=11,2 IC95\%1,5$84,0 ; \mathrm{p}=0,002)$ no pós-operatório, quando comparados aos sem risco nutricional. Não houve associação do risco de sarcopenia com a presença de complicaçóes infecciosas e óbito ao longo do período pós-operatório ( $p>0,05)$. Conclusáo: Os pacientes oncológicos em risco nutricional foram aqueles que apresentaram maior risco de complicaçôes infecciosas e de óbito no pós-operatório, quando comparados aos sem risco nutricional ou em risco de sarcopenia.

Palavras-chave: Oncologia Cirúrgica; Estado Nutricional; Sarcopenia; Complicações Pós-Operatórias; Mortalidade.

\section{ABSTRACT}

Introduction: Surgical patients who are at either nutritional or sarcopenia risk may have worst outcomes in the postoperative period. Objective: To investigate whether nutritional or sarcopenia risk is associated with mortality and postoperative complications in cancer patients undergoing major operations. Method: Prospective cohort bicentrical study enrolling 220 adult oncological patients submitted to major surgeries at Cancer Hospital and Santa Casa de Misericordia in Cuiabá-MT. Patients were classified with or without nutritional risk per the Nutritional Risk Screening 2002 and sarcopenia risk according to the Strength, Assistance with walking, Rise from a chair, Climb stairs - and Falls questionnaire preoperatively. The outcomes variables were postoperative infectious complications and death. Results: Patients with nutritional risk showed higher risk of infectious complications ( 24.6 vs. $5.1 \%$; $R R=4.8$ CI95\% 1.94-12; $\mathrm{p}<0.001$ ) or die (11.5 vs. $1.0 \% ; \mathrm{RR}=11.2 \mathrm{CI} 95 \% 1.5-84.0 ; \mathrm{p}=0.002)$ in post-operation when compared to patients without nutritional risk. There was no association between sarcopenia risk with infectious complications or mortality during post-operation ( $p>0.05)$. Conclusion: Oncological patients with nutritional risk have higher risk of developing postoperative infectious complications or die when compared with patients without nutritional risk or in risk of sarcopenia.

Key words: Surgical Oncology; Nutritional Status; Sarcopenia; Postoperative Complications; Mortality.

\section{RESUMEN}

Introducción: Los pacientes de cáncer quirúrgico con riesgo nutricional o de sarcopenia pueden evolucionar con peores resultados en el postoperatorio. Objetivo: Investigar si existe una asociación entre el riesgo nutricional y la sarcopenia con complicaciones y mortalidad en el postoperatorio de pacientes con cáncer sometidos a operaciones mayores. Método: Estudio prospectivo de cohorte bicéntrico realizado con 220 pacientes adultos con cáncer que se sometieron a operaciones importantes en el Hospital de Cáncer y Santa Casa de Misericordia en Cuiabá, Mato Grosso. La muestra estudiada se clasificó con o sin riesgo nutricional por Nutritional Risk Screening 2002 y sarcopenia de acuerdo con el cuestionario Strength, Assistance with walking, Rise from a chair, Climb stairs - and Falls, en el pre operatorio. Las variables de resultado fueron complicaciones infecciosas y muerte en la postoperatorio. Resultados: Los pacientes con riesgo nutricional mostraron un mayor riesgo de complicaciones infecciosas (24,6 vs. 5,1\%; RR=4,8 IC95\% 1,94-12; $\mathrm{p}<0,001)$ y muerte $(11,5$ vs. $1,0 \%$; $\mathrm{RR}=11,2 \mathrm{IC} 95 \% 1,5-84,0 ; \mathrm{p}=0,002)$ en la postoperatorio en comparación con aquellos sin riesgo nutricional. Sin embargo, no hubo asociación entre el riesgo de sarcopenia y la presencia de complicaciones infecciosas y muerte durante el período postoperatorio ( $p>$ 0.05 ). Conclusión: Los pacientes con cáncer en riesgo nutricional según NRS-2002, fueron aquellos que tenían un mayor riesgo de complicaciones infecciosas y muerte en la postoperatorio, en comparación con aquellos sin riesgo nutricional o con riesgo de sarcopenia.

Palabras clave: Oncología Quirúrgica; Estado Nutricional; Sarcopenia; Complicaciones Postoperatorias; Mortalidad.

\footnotetext{
1-5,7,8 Universidade Federal de Mato Grosso. Faculdade de Medicina. Programa de Pós-Graduação em Ciências da Saúde. Cuiabá (MT), Brasil. ${ }^{6,7}$ Centro Universitário de Várzea Grande (Univag). Várzea Grande (MT), Brasil.

'E-mail: hadassanovaes16@gmail.com. Orcid iD: https://orcid.org/0000-0002-3553-4999; ${ }^{2}$ E-mail: maristelaluftp@gmail.com. Orcid iD: https://orcid.org/0000-00024657-5219; ${ }^{3}$ E-mail: thayse.emanuelli@hotmail.com. Orcid iD: https://orcid.org/0000-0002-7835-9318; ${ }^{4}$ E-mail: antawara512@gmail.com. Orcid iD: https://orcid. org/0000-0002-2391-9509; ${ }^{5}$ E-mail: andreolene@gmail.com. Orcid iD: https://orcid.org/0000-0002-1223-7625; ${ }^{6}$ E-mail: marianaborgesthe@hotmail.com. Orcid iD: https://orcid.org/0000-0003-2945-5570; ${ }^{7}$ E-mail: aguilar@terra.com.br. Orcid iD: https://orcid.org/0000-0002-3583-6612; ${ }^{8}$ E-mail: dianadock@hotmail.com. Orcid iD: https://orcid.org/0000-0003-0205-6676

Endereço para correspondência: Diana Borges Dock-Nascimento. Rua Quarenta e Nove, 2367 - Boa Esperança. Cuiabá (MT), Brasil. CEP 78.060-900. E-mail: dianadock@hotmail.com
} 


\section{INTRODUÇÃO}

Há quase nove décadas, Studley ${ }^{1}$ documentou que a perda ponderal estava associada ao aumento da mortalidade em pacientes cirúrgicos. Atualmente, no Brasil, cerca de $42 \%$ dos pacientes oncológicos internados apresentam perda de peso, e $45 \%$ internam desnutridos ou em risco de desnutrição ${ }^{2}$. Entretanto, entre os pacientes com tumores de cavidade oral, esôfago e estômago, a desnutrição gira entre $62 \%$ e $84 \%{ }^{2}$.

Nesse contexto, os prejuízos na condição nutricional têm sido constantemente associados a piores desfechos clínicos, os quais refletem na resposta ao tratamento oncológico, no aumento de complicaçóes, nas taxas de óbito e nos custos hospitalares ${ }^{3-5}$. Especificamente em relação ao risco nutricional, $30 \%$ a $70 \%$ dos pacientes internam com esse diagnóstico ${ }^{4,5}$, o que está significativamente associado ao aumento de complicaçóes pós-operatórias $s^{5-8}$. Nesse sentido, um estudo mostrou que pacientes submetidos à gastrectomia radical por câncer, que estavam em risco nutricional, apresentaram um aumento nas taxas de complicaçóes pós-operatórias e óbito ${ }^{6}$.

A sarcopenia, principalmente na última década, passou a ser associada a prejuízos na evoluçáo dos pacientes no período pós-operatório ${ }^{9-11}$. A síndrome sarcopênica é caracterizada pela perda progressiva e generalizada da função e da massa muscular esquelética, levando à diminuiçăo da condição funcional e da performance ${ }^{9-11}$. Particularmente, entre os pacientes oncológicos, a sarcopenia é um recente preditor de aumento de tempo de hospitalização, reinternações, complicaçôes infecciosas e mortalidade ${ }^{9-13}$.

Portanto, dentro dos cuidados perioperatórios, é importante uma ampliação do rastreio com a realização da triagem de risco nutricional e da sarcopenia ${ }^{11-15}$. Entretanto, apesar de a prática da triagem de risco nutricional e da sarcopenia serem amplamente aceitas e conhecidas, até o momento, nenhum estudo investigou, ao mesmo tempo, na mesma populaçáo de pacientes oncológicos, qual desses dois riscos apresentam melhor ou maior associação com pior prognóstico no pós-operatório. Desta forma, a equipe poderá intervir mais rapidamente por meio dos cuidados multimodais de aceleração da recuperação no paciente cirúrgico ${ }^{16}$.

Com base no exposto, foi conduzido um estudo prospectivo com o objetivo de determinar a associação entre o risco nutricional e o de sarcopenia com complicaçóes e mortalidade no pós-operatório de pacientes oncológicos submetidos a cirurgias de grande porte.

\section{MÉTODO}

Trata-se de um estudo bicêntrico, de coorte prospectivo, realizado no período de julho de 2018 a abril de 2019, com pacientes oncológicos adultos (idade $\geq 18$ anos), admitidos no Hospital de Câncer e na Santa Casa de Misericórdia de Cuiabá-MT. Os pacientes que aceitaram participar do estudo assinaram um Termo de Consentimento Livre e Esclarecido (TCLE). A investigação foi aprovada pelo Comitê de Ética em Pesquisa da Universidade Federal de Mato Grosso (UFMT), número 2.666.168/2018.

Foram incluídos pacientes adultos com câncer, candidatos a operaçóes oncológicas de grande porte. Definiu-se cirurgia de grande porte aquela com maior probabilidade de perda de fluido e sangue, de acordo com parecer número 006/2015 do Conselho Federal de Medicina ${ }^{17}$. Os pacientes diagnosticados com neoplasia de pele não melanoma, doença avançada, e os que se recusaram a assinar o TCLE ou não quiseram participar da pesquisa, em qualquer fase do estudo, foram excluídos. Excluíram-se também os pacientes cujos dados foram perdidos, ou que tiveram suas cirurgias suspensas por qualquer motivo, ou foram transferidos no pós-operatório para outro hospital.

Os pacientes foram classificados com ou sem risco nutricional e de sarcopenia, no pré-operatório, conforme definição a seguir. As variáveis principais foram a ocorrência de complicações infecciosas no pós-operatório e óbito. Como covariáveis, registraram-se idade (considerou-se idoso pacientes com idade $\geq 60$ anos), sexo, índice de massa corporal $\left(\mathrm{Kg} / \mathrm{m}^{2}\right)$, escore American Society of Anesthesiologists (ASA), tipo de operação segundo a localização do tumor, tempo de operação (minutos) e de internação (em dias; até a alta ou óbito). Os dados foram coletados no pré-operatório imediato; ou seja, cerca de uma a duas horas antes da operação, e no pós-operatório até alta ou óbito hospitalar.

Para determinar o risco nutricional, foi utilizada a ferramenta Nutritional Risk Screening 2002 (NRS-2002). Os pacientes com pontuação $\geq 3$ foram classificados como sendo de risco nutricional ${ }^{5,18}$. Para a avaliaçáo do risco de sarcopenia, foi utilizado o questionário Strength, Assistance with walking, Rise from a chair, Climb stairs - and Falls $(\mathrm{SARC}-\mathrm{F})^{11,19}$. Esse questionário é composto por cinco perguntas que avaliam força, caminhada, levantar-se de uma cadeira, subir escadas e o histórico de quedas. As pontuaçóes variam de 0 a 10 pontos, sendo de $0-2$ pontos para cada item ${ }^{11}$. Os pacientes com pontuação $\geq 4$ pontos foram classificados como em risco de sarcopenia $(\text { SARC-F } \geq 4)^{11,19}$.

Consideraram-se complicaçôes infecciosas: presença de pneumonia, infecção no sítio cirúrgico, deiscências de anastomose ou parede, infecção do trato urinário e sepse. Todas as definiçôes de complicaçôes infecciosas foram citadas em outros artigos publicados pelo mesmo grupo $^{20,21}$. As complicações foram também classificadas pelos critérios de Clavien-Dindo ${ }^{22}$. 
Inicialmente, foi aplicado o teste de Kolmogorov-Smirnov para determinar a normalidade dos dados contínuos. Os dados normalmente distribuídos foram apresentados em médias e desvios-padrão e os distribuídos de maneira não normal foram apresentados em mediana e intervalo interquartil (M;IIQ). Utilizou-se o teste do qui-quadrado (risco relativo e intervalo de confiança de 95\%) para determinar a associaçáo do risco nutricional e de sarcopenia com a presença de complicaçóes infecciosas e óbito. O teste de Mann-Whitney foi utilizado para comparar o tempo de internação em dias (distribuição não normal) entre os pacientes com e sem risco nutricional ou de sarcopenia. Estabeleceu-se um limite de significância estatística de $5 \%(\mathrm{p}<0,05)$. Para a análise estatística, foi utilizado o Programa Statistical Package for the Social Sciences 20.0 (SPSS Statistics; IBM, Armonk, NY, USA).

\section{RESULTADOS}

Foram elegíveis 338 pacientes, dos quais, 12 foram excluídos por apresentarem câncer de pele não melanoma, 14 por doença avançada, 13 por perda de dados e 79 por suspensão da operaçáo. Participaram do estudo 220 pacientes submetidos a cirurgias de grande porte, com média de idade de $58,7 \pm 14,0$ anos. Os demais dados clínicos e demográficos estão descritos na Tabela 1.

Tabela 1. Características clínicas e demográficas dos pacientes estudados $(n=220)$

\begin{tabular}{|c|c|}
\hline Variáveis & Valores \\
\hline Idoso (n; \%) & $111(50,5)$ \\
\hline \multicolumn{2}{|l|}{$\operatorname{Sexo}(n ; \%)$} \\
\hline Feminino & $109(49,5)$ \\
\hline Masculino & $111(50,5)$ \\
\hline$I M C\left(\mathrm{~kg} / \mathrm{m}^{2}\right)(M ; I I Q)$ & $26,1(23,0-30,1)$ \\
\hline Escore ASA I e II (n; \%) & $202(91,8)$ \\
\hline \multicolumn{2}{|l|}{$\begin{array}{l}\text { Tipo de operação conforme } \\
\text { local do tumor }\end{array}$} \\
\hline - Urológico & $86(39,1)$ \\
\hline - Trato digestório & $69(31,4)$ \\
\hline - Mama & $29(13,2)$ \\
\hline - Cabeça e pescoço & $17(7,7)$ \\
\hline - Outros & $19(8,6)$ \\
\hline Tempo de cirurgia (minutos) (M; IIQ) & $125(90-205)$ \\
\hline Tempo de internação (dias) (M; IQ) & $3(2-7)$ \\
\hline
\end{tabular}

Legendas: IMC: índice de massa corporal $\left(\mathrm{Kg} / \mathrm{m}^{2}\right)$; ASA: American Society of Anesthesiologists.

Nota: Valores expressos em: média e desvio-padrão $(\mathrm{M} \pm \mathrm{DP})$; número e percentual ( $\mathrm{n}$ \% \%); mediana e intervalo interquartil (M; IIQ) de acordo com a distribuiçáo dos dados.
A Figura 1 apresenta os resultados de risco nutricional e risco de sarcopenia no pré-operatório.

No pós-operatório, $37,7 \%(\mathrm{n}=83)$ dos pacientes apresentaram pelo menos uma complicação, sendo $15,9 \%(\mathrm{n}=35)$ infecciosas. Também, a maioria dos casos complicados foi de natureza leve, sendo $61(27,7 \%)$ classificados como Clavien-Dindo I ou II. Quinze pacientes $(6,8 \%)$ foram a óbito no pós-operatório.

Os pacientes em risco nutricional foram aqueles que permaneceram mais tempo internados do que os sem risco nutricional [5,0(3-9) vs. 2(1-3); $\mathrm{p}<0,001]$. Não houve diferença no tempo em dia de internação entre os pacientes com e sem risco de sarcopenia $[4(1-8) v s$. $3(2-7) ; \mathrm{p}=0,781]$.

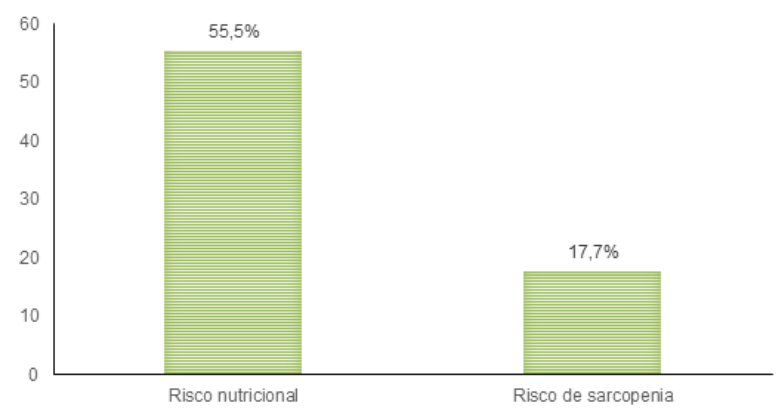

Figura 1. Distribuição do risco nutricional e de sarcopenia entre os pacientes estudados

De acordo com a Tabela 2, os pacientes em risco nutricional mostraram maior risco de complicaçôes infecciosas $(24,6$ vs. $5,1 \%$; RR=4,8 IC95\%1,94-12; $\mathrm{p}<0,001)$ e de óbito ( 11,5 vs. $1,0 \%$; RR= 11,2 IC95\% 1,5$84,0 ; \mathrm{p}=0,002)$ no pós-operatório quando comparados aos sem risco nutricional. Não houve associação do risco de sarcopenia com a presença de complicaçóes infeciosas $(23,1$ vs. $14,4 \%$; RR=1,61 IC95\% 0,82-3,15; $\mathrm{p}=0,177)$ ou óbito $(10,3$ vs. 6,1\%; RR=1,69 IC095\% 0,58-5,02; $\mathrm{p}=0,348$ ) ao longo do pós-operatório (Tabela 2).

\section{DISCUSSÃO}

O risco nutricional diagnosticado pela NRS-2002 mostrou uma significativa associação com o risco de complicação infecciosa e com a mortalidade pós-operatória. O risco de complicaçóes infecciosas, entre os pacientes com risco nutricional, aumentou quase cinco vezes e o risco de óbito em mais de 11 vezes, quando comparados aos pacientes sem risco nutricional. Também, os pacientes em risco nutricional foram aqueles que ficaram três dias a mais internados no pós-operatório quando comparados aos sem risco nutricional. 
Tabela 2. Associação do risco nutricional e de sarcopenia com complicações infecciosas e óbito

\begin{tabular}{lllll}
\hline \multirow{2}{*}{ Evento } & Risco nutricional & \multicolumn{3}{c}{ Risco de sarcopenia } \\
\cline { 2 - 5 } & $\mathbf{R R}(\mathbf{I C 9 5 \% )}$ & $\mathbf{P}$ & $\mathbf{R R}$ (IC95\%) & $\mathbf{P}$ \\
\hline Complicações infecciosas & $4,8(1,94-12)$ & $<0,001$ & $1,61(0,82-3,15)$ & 0,177 \\
Óbito & $11,2(1,5-84,0)$ & 0,002 & $1,69(0,58-5,02)$ & 0,348 \\
\hline
\end{tabular}

Legendas: Teste do qui-quadrado; RR: risco relativo; IC95\%: intervalo de confiança de 95\%.

Os mesmos desfechos não foram encontrados ao estudar os pacientes em risco de sarcopenia. Nessa linha, o último Consenso Europeu EWGSOP2 (European Working Group on Sarcopenia in Older People $)^{11}$ recomenda o uso do questionário SARC-F para o rastreio da sarcopenia em pacientes com suspeita clínica. Esse questionário é útil, simples, de baixo custo, e facilmente aplicável ${ }^{11,19}$; contudo, não se encontrou associação do risco de sarcopenia, diagnosticada pelo questionário SARC-F, com a ocorrência de complicaçôes infeciosas e óbito no pós-operatório, quando comparados aos pacientes com risco nutricional. Isso pode ter sido influenciado pelo percentual de pacientes que estavam em risco nutricional e de sarcopenia.

O próprio Consenso Europeu ${ }^{11}$ cita que o SARC-F é uma ferramenta que diagnostica casos mais graves de alteração da força muscular ${ }^{23}$. Ainda acrescenta que esse questionário apresenta uma sensibilidade de moderada a baixa e uma alta especificidade ${ }^{11}$. Neste sentido, um artigo recente desse grupo mostrou que pacientes em risco de sarcopenia, pelo SARC-F, que apresentavam também baixa força de preensão palmar, foram os que se comportaram com maior risco de complicaçóes infecciosas no pós-operatório ${ }^{24}$. Também, Barbosa-Silva et al. ${ }^{25}$, em 2016, mostraram que a avaliação do SARC-F combinado com a circunferência da panturrilha (SARC-CalF) foi mais sensível para detectar pacientes em risco de sarcopenia quando comparados ao SARC-F isolado ( $\mathrm{p}=0,027)$.

De acordo com a ferramenta NRS-2002, havia três vezes mais pacientes em risco nutricional do que em risco de sarcopenia no pré-operatório imediato. Nota-se que as duas ferramentas de triagem estudadas revelaram valores bem distintos do risco que cada uma expressa; ou seja, $55,5 \%$ e $17,7 \%$. De acordo com uma metanálise realizada com 12.800 pacientes, o questionário SARC-F apresentou uma baixa sensibilidade para o rastreamento da sarcopenia, embora tenha apresentado uma alta especificidade ${ }^{26}$.

Portanto, o SARC-F parece ser uma ferramenta eficaz para selecionar pacientes que devem ser submetidos a mais testes para confirmar o diagnóstico de sarcopenia ${ }^{26}$. Neste estudo, esse resultado foi uma surpresa, uma vez que o SARC-F avalia as condiçóes relacionadas à capacidade funcional e à performance muscular ${ }^{11,19,26,27}$, que se modificam rapidamente antecedendo às alterações antropométricas ${ }^{28-31}$, o que permite diagnosticar mais precocemente os prejuízos na condição do paciente ${ }^{11,12}$ e, ainda, a associação com piores desfechos. Por outro lado, o NRS-2002 é um instrumento preconizado pela Sociedade Europeia de Nutriçáo Clínica e Metabolismo (ESPEN) para triagem nutricional, e tem sido empregado com frequência para associar o risco nutricional e as complicações pós-operatórias ${ }^{9,10}$.

Estudos anteriores mostraram que a NRS-2002 é uma excelente ferramenta de triagem para diagnosticar o risco nutricional, o que corrobora os dados encontrados nesta investigação ${ }^{4-8}$. Diferentemente do questionário SARC-F, a NRS-2002 foi desenvolvida para identificar pacientes que podem se beneficiar de uma intervenção nutricional; ou seja, aqueles que apresentam perda de peso, baixo índice de massa corporal, reduçáo da ingestão alimentar, idade avançada e/ou um aumento do estresse metabólico relacionado à condição clínica ou cirúrgica ${ }^{18}$.

Todos esses dados que compóem a NRS-2002 podem, em conjunto, terem contribuído para os resultados aqui encontrados. Neste sentido, um estudo publicado em 2018 mostrou que pacientes com câncer retal, em risco nutricional, foram aqueles que apresentaram um estágio mais avançado da doença, maior escore ASA $(\mathrm{OR}=2,4)$, maior tempo de operação $(\mathrm{OR}=1,97)$ e maior escore da NRS-2002 (OR=2,04), quando comparados aos pacientes sem risco nutricional ${ }^{8}$. Os autores concluíram que a presença do risco nutricional, determinada pela NRS-2002, é fator de risco independente para pacientes cirúrgicos com câncer ${ }^{8}$. Uma questáo que deve ser salientada é que, comparada a outras ferramentas de triagem, a NRS-2002 permite uma gradaçáo do efeito da doença (escore 1 a 3) e isto já coloca o avaliador em posição de pontuar os pacientes com risco nutricional em razáo da gravidade da doença ${ }^{8}$.

Para exemplificar, a condiçáo de ser um paciente agendado para uma operaçáo de grande porte, já revela um índice de estresse e gravidade muito importante para o risco de desnutriçãa ${ }^{8,18}$, isto certamente colaborou para o maior percentual de pacientes que estavam em risco de desnutrição. Também, outra reflexão que se faz necessária, sobre a coleta de dados para definir o diagnóstico da presença ou náo do risco.

A pontuação obtida pelo SARC-F foi realizada por meio de cinco perguntas ${ }^{11}$. Esta foi concluída de forma muito subjetiva, conforme a resposta do paciente; ou seja, 
o escore final denota a interpretação do paciente ${ }^{11,19}$. Isso muito se difere da NRS-2002, uma vez a pontuação se refere a critérios objetivos e técnicos, conforme a condição avaliada por profissional treinado e não pelo julgamento do paciente $e^{7,8}$. Em consonância com estes resultados, uma metanálise realizada com 3.527 pacientes, submetidos a cirurgias abdominais, mostrou que os pacientes em risco nutricional, de acordo com a NRS-2002, apresentaram um aumento nas taxas de complicações, na mortalidade e no tempo de internação ${ }^{8}$.

Os autores enfatizaram que é amplamente assumido que o estado nutricional pré-operatório é determinante para os desfechos pós-operatórios em pacientes submetidos a operaçôes de grande porte. E ainda que vários aspectos das deficiências nutricionais podem levar à desnutrição ${ }^{8}$. Embora muitos estudos tenham mostrado que pacientes em risco nutricional ou de sarcopenia são os mais graves e evoluem com piores desfechos, nenhum estudo comparou, até o momento, esses dois escores de triagem em uma mesma população de pacientes ${ }^{5-10}$. Assim, esses resultados podem contribuir para outras reflexóes em relação à qual triagem deve ser utilizada na prática clínica pré-operatória.

Convém frisar que ambas as ferramentas de triagem são simples e rápidas e podem ser realizadas à beira do leito com o paciente deitado ou sentado, o que otimiza o rastreamento, possibilitando intervençóes precoces para reduzir complicaçóes e custos ${ }^{5,8,11,14}$. Entretanto os dados deste estudo mostraram que apenas o risco nutricional pela NRS-2002 foi capaz de predizer desfechos desfavoráveis no pós-operatório. Por isso, para esses pacientes, a intervenção nutricional precoce, com o objetivo de pré-habilitar o paciente, para a operação eletiva, deve ser incorporada aos cuidados perioperatórios.

A evidência reforça que os pacientes em risco de desnutrição, pela NRS 2002 e que recebem uma terapia nutricional, antes da operação, mostram uma aceleração na recuperação pós-operatório com menor ocorrência de eventos adversos ${ }^{16}$. Em 2003, Kondrup et al. ${ }^{18}$ mostraram que os pacientes cirúrgicos, que apresentaram risco nutricional maior igual a três pontos, foram aqueles que, significativamente, apresentaram maior benefício com a terapia nutricional, diferente daqueles com escore menor que três pontos.

Por conseguinte, cabe ressaltar que os projetos multimodais, de cuidados perioperatórios como a Aceleração da Recuperação Total Pós-operatória (ACERTO), preconizam, dentro das açóes proativas, a realização da triagem de risco nutricional, bem como o início da pré-habilitação nutricional para os pacientes em risco nutricional ${ }^{16}$. Embora os resultados aqui encontrados tragam mais discussóes sobre o rastreamento do paciente cirúrgico eletivo oncológico, estes devem ser analisados com cautela. A amostra apresentava uma heterogeneidade segundo o tipo e a localização do tumor.

O tamanho da amostra também pode ter influenciado nos resultados encontrados principalmente no percentual de pacientes que estavam em risco de sarcopenia. Ainda, a metade dos pacientes era de idosos. Contudo, em virtude da expressiva quantidade de operaçóes de grande porte, que ocorrem entre os pacientes oncológicos, o rastreio nutricional é determinante para a reduçáo da morbidade operatória e dos custos com o paciente cirúrgico ${ }^{16,21}$.

\section{CONCLUSÃO}

Em síntese, pôde-se concluir que os pacientes oncológicos em risco nutricional diagnosticados pela NRS-2002 foram aqueles que apresentaram maior risco de complicaçóes infecciosas e de óbito quando comparados aos sem risco nutricional. O diagnóstico do risco de sarcopenia, pelo questionário SARC-F, não foi capaz de mostrar essas associaçóes em pacientes oncológicos submetidos a operaçóes de grande porte.

\section{CONTRIBUIÇÕES}

Todos os autores contribuíram na concepção e/ ou no planejamento do estudo; na obtençáo, análise e interpretação dos dados; assim como na redação e revisão crítica; e aprovaram a versão final a ser publicada.

\section{DECLARAÇÃO DE CONFLITO DE INTERESSES}

Nada a declarar.

\section{FONTES DE FINANCIAMENTO}

Não há.

\section{REFERÊNCIAS}

1. Studley HO. Percentage of weight loss: basic indicator of surgical risk in patients with chronic peptic ulcer. JAMA. 1936;106(6):458-60. doi: http://doi.org/10.1001/ jama.1936.02770060032009

2. Instituto Nacional de Câncer José Alencar Gomes da Silva. Inquérito brasileiro de nutrição oncológica [Internet]. Rio de Janeiro: INCA; 2013 [acesso 2020 abr 23]. Disponível em: https:/www.inca.gov.br/sites/ ufu.sti.inca.local/files//media/document//inqueritobrasileiro-nutricao-oncologica.pdf

3. Ravasco P. Nutrition in cancer patients. J Clin Med. 2019;8(8):1211. doi: http://doi.org/10.3390/ jcm 8081211

4. Sun DL, Li WM, Li SM, et al. Impact of nutritional support that does and does not meet guideline standards 
on clinical outcome in surgical patients at nutritional risk: a prospective cohort study. Nutr J. 2016;15(1):78. doi: http://doi.org/10.1186/s12937-016-0193-6

5. Wang X, Wu J, Lei S, et al. Effect of preoperative nutritional risk screening on postoperative recovery in patients with laparoscopic-assisted radical resection for colorectal cancer. Gastroenterol Res Pract. 2020;2020:2046253. doi: http://doi.org/10.1155/2020/2046253

6. Zhuang CL, Wang SL, Huang DD, et al. Risk factors for hospital readmission after radical gastrectomy for gastric cancer: a prospective study. PLoS One. 2015;10(4):e0125572. doi: http://doi.org/10.1371/ journal.pone.0125572

7. Guo W, Ou G, Li X, et al. Screening of the nutritional risk of patients with gastric carcinoma before operation by NRS 2002 and its relationship with postoperative results. J Gastroenterol Hepatol. 2010;25(4):800-3. doi: http:// doi.org/10.1111/j.1440-1746.2009.06198.x

8. Sun Z, Kong XJ, Jing X, et al. Nutritional risk screening 2002 as a predictor of postoperative outcomes in patients undergoing abdominal surgery: a systematic review and meta-analysis of prospective cohort studies. PLoS One. 2015;10(7):e0132857. doi: http://doi.org/10.1371/ journal.pone. 0132857

9. Zhou CJ, Zhang FM, Zhang FY, et al. Sarcopenia: a new predictor of postoperative complications for elderly gastric cancer patients who underwent radical gastrectomy. J Surg Res. 2017;211:137-46. doi: http:// doi.org/10.1016/j.jss.2016.12.014

10. Simonsen C, de Heer P, Bjerre ED, et al. Sarcopenia and postoperative complication risk in gastrointestinal surgical oncology: a meta-analysis. Ann Surg. 2018;268(1):58-69. doi: http://doi.org/10.1097/ SLA.0000000000002679

11. Cruz-Jentoft AJ, Bahat G, Bauer J, et al. Sarcopenia: revised European consensus on definition and diagnosis. Age Ageing. 2019;48(1):16-31. doi: http://doi. org/10.1093/ageing/afy169

12. Xu J, Zheng B, Zhang S, et al. Effects of preoperative sarcopenia on postoperative complications of minimally invasive oesophagectomy for oesophageal squamous cell carcinoma. J Thorac Dis. 2019;11(6):2535-45. doi: http://doi.org/10.21037/jtd.2019.05.55

13. Ryan AM, Power DG, Daly L, et al. Cancer-associated malnutrition, cachexia and sarcopenia: the skeleton in the hospital closet 40 years later. Proc Nutr Soc. 2016;75(2):199-211. doi: http://doi.org/10.1017/ S002966511500419X

14. Bauer J, Morley JE, Schols AMWJ, et al. Sarcopenia: a time for action. An SCWD position paper. J Cachexia Sarcopenia Muscle. 2019;10(5):956-61. doi: http://doi. org/10.1002/jcsm. 12483

15. Fearon K, Strasser F, Anker SD, et al. Definition and classification of cancer cachexia: an international consensus. Lancet Oncol. 2011;12(5):489-95. doi: http://doi.org/10.1016/S1470-2045(10)70218-7

16. de-Aguilar-Nascimento JE, Salomão AB, Waitzberg DL, et al. ACERTO guidelines of perioperative nutritional interventions in elective general surgery. Rev Col Bras Cir. 2017;44(6):633-48. doi: https://doi.org/10.1590/010069912017006003

17. Conselho Federal de Medicina (BR). Parecer consulta no 006, de 28 de abril de 2015. Ementa: Competência do Pediatra para atendimento inicial à criança. $\mathrm{Na}$ falta desse profissional, o médico clínico deve prestar o primeiro atendimento e encaminhar o paciente para um atendimento especializado [Internet]. [acesso 2020 ago 08]. Disponível em: http://www.portalmedico.org.br/ pareceres/crmes/pareceres/2015/0006_2015.pdf

18. Kondrup J, Allison SP, Elia M, et al. ESPEN guidelines for nutrition screening 2002. Clin Nutr. 2003;22(4):415-21. doi: https://doi.org/10.1016/s0261-5614(03)00098-0

19. Malmstrom TK, Morley JE. Sarcopenia: the target population. J Frailty Aging. 2013;2(1):55-6. doi: https:// doi.org/10.14283/jfa.2013.8

20. Aguilar-Nascimento JE, Marra JG, Slhessarenko $\mathrm{N}$, et al. Efficacy of National Nosocomial Infection Surveillance score, acute-phase proteins, and interleukin-6 for predicting postoperative infections following major gastrointestinal surgery. Sao Paulo Med J. 2007;125(1):34-41. doi: https://doi.org/10.1590/ S1516-31802007000100007

21. Bicudo-Salomão A, Salomão RF, Cuerva $\mathrm{MP}$, et al. Factors related to the reduction of the risk of complications in colorectal surgery within perioperative care recommended by the ACERTO protocol. ABCD Arq Bras Cir Dig. 2019;32(4):e1477. doi: https://doi. org/10.1590/0102-672020190001e1477

22. Moreira LF, Pessôa MCM, Mattana DS, et al. Adaptaçáo cultural e teste da escala de complicaçóes cirúrgicas de Clavien-Dindo traduzida para o Português do Brasil. Rev Col Bras Cir. 2016;43(3):141-8. doi: https:/doi. org/10.1590/0100-69912016003001

23. Bahat G, Yilmazi O, Kiliç C et al. Performance of SARC-F in regard to sarcopenia definitions, muscle mass and functional measures. J Nutr Health Aging. 2018. 22:898-903. doi: https://doi.org/10.1007/s12603-0181067-8

24. Behne TEG, Dock-Nasimento DB, Sierra JC, et al. Association between preoperative potential sarcopenia and survival of cancer patients undergoing major surgical procedures. Rev Col Bras Cir. 2020;47:e20202528. doi: http://doi.org/10.1590/0100-6991e-20202528

25. Barbosa-Silva TG, Menezes AMB, Bielemann RM, et al. Enhancing SARC-F: improving sarcopenia screening in the clinical practice. J Am Med Dir Assoc. 2016;17(12):1136-41. doi: http://doi.org/10.1016/j. jamda.2016.08.004 
26. Ida S, Kaneko R, Murata K. SARC-F for screening of sarcopenia among older adults: a meta-analysis of screening test accuracy. J Am Med Dir Assoc. 2018;19(8):685-9. doi: http://doi.org/10.1016/j. jamda.2018.04.001

27. Malmstrom TK, Miller DK, Simonsick EM, et al. SARC-F: a symptom score to predict persons with sarcopenia at risk for poor functional outcomes. J Cachexia Sarcopenia Muscle. 2016;7(1):28-36. doi: http://doi.org/10.1002/jcsm.12048

28. Humphreys J, de la Maza P, Hirsch S, et al. Muscle strength as a predictor of loss of functional status in hospitalized patients. Nutrition. 2002;18(7-8):616-20. doi: http://doi.org/10.1016/s0899-9007(02)00756-6

29. Gava MG, Castro-Barcellos HM, Caporossi C, et al Enhanced muscle strength with carbohydrate supplement two hours before open cholecystectomy: a randomized, double-blind study. Rev Col Bras Cir. 2016;43(1):54-9. doi: https://doi.org/10.1590/0100-69912016001011

30. Correa-Arruda WS, Vaez IA, Aguilar-Nascimento JE, et al. Effects of overnight fasting on handgrip strength in inpatients. Einstein (Sao Paulo). 2019;17(1): eAO4418. doi: https://doi.org/10.31744/einstein journal/2019ao4418

31. Arora NS, Rochester DF. Effect of body weight and muscularity on human diaphragm muscle mass, thickness, and area. J Appl Physiol Respir Environ Exerc Physiol. 1982;52(1):64-70. doi: https://doi.org/10.1152/ jappl.1982.52.1.64 